\title{
THE PHYSICS OF QUANTUM WELL INFRARED PHOTODETECTORS
}




\section{SERIES IN MODERN CONDENSED MATTER PHYSICS}

\section{Editors-in-charge: I. Dzyaloshinski, S. Lundqvist and Yu Lu}

\section{Published}

Vol. 1: Exotic Properties of Superfluid Helium 3 by G. E. Volovik

Vol. 2: Quantum Dissipative Systems by $U$. Weiss

Vol. 4: Simple Views on Condensed Matter by P. G. de Gennes

\section{Forthcoming}

Modulated Structures : Commensurate, incommensurate, Stochastic Structures and Quasicrystals by V. Pokrovsky and G. Uimin Lectures on Electron Correlation \& Magnetism by $P$. Fazekas Low-Dimensional Quantum Field Theories for Condensed Matter Physicists eds. Y. Lu, S. Lundqvist and G. Morandi 
seriesin

Mocom

Condensed

Mattor

Bhystes

Vol. 7

\section{THE PHYSICS OF QUANTUM WELL INFRARED PHOTODETECTORS}

\section{K. K. Choi}

US Army Research Laboratory, Maryland 
Published by

World Scientific Publishing Co. Pte. Ltd.

P O Box 128, Farrer Road, Singapore 912805

USA office: Suite 1B, 1060 Main Street, River Edge, NJ 07661

UK office: 57 Shelton Street, Covent Garden, London WC2H 9HE

\section{Library of Congress Cataloging-in-Publication Data}

Choi, K. K.

The physics of quantum well infrared photodetectors / K. K. Choi,

p. cm. -- (Series in modern condensed matter physics ; vol. 7)

Includes bibliographical references and index.

ISBN 9810228724

1. Photon detectors. 2. Photoelectronic devices. 3. Quantum wells.

4. Infrared detectors. I. Title. II. Series.

QC787.P46C46 1997

621.36 '72--dc21

$97-15121$

CIP

\section{British Library Cataloguing-in-Publication Data}

A catalogue record for this book is available from the British Library.

Copyright $(\mathcal{C} 1997$ by World Scientific Publishing Co. Pte. Ltd.

All rights reserved. This book, or parts thereof, may not be reproduced in any form or by any means, electronic or mechanical, including photocopying, recording or any information storage and retrieval system now known or to be invented, without written permission from the Publisher.

For photocopying of material in this volume, please pay a copying fee through the Copyright Clearance Center, Inc., 222 Rosewood Drive, Danvers, MA 01923, USA. In this case permission to photocopy is not required from the publisher.

Printed in Singapore 


\section{Preface}

Although it was already well known that the energy-level spacing in a quantum well (QW) structure can be tailored to the infrared photon energy, the early attempt of using $\frac{6}{\mathrm{C}} \mathrm{QWs}$ in infrared detection was based on free electron absorption in QWs to bring the Electrons over the top of the barriers. The energy level structure and the large dipole

政 detectors is expected to be low.

In 1985, West and Eglash observed strong intersubband (envelope wavefunction) transition in $\mathrm{GaAs} / \mathrm{Al}_{x} \mathrm{Ga}_{1-x}$ As multiple quantum wells (MQW) that initiated serious investigations in using MQWs as infrared photodetectors. In 1987, Choi et al. and Levine et al. published a series of papers illustrating the basic operating principles of the detector, and demonstrating sensitive infrared detection based on this transition. In these MQW structures, the final QW state of transition was placed either slightly above or below the top of the barriers to obtain optimum sensitivity. However, since the barriers in these structures were relatively thin, the sensitivity of the detectors did not improve significantly at a lower operating temperature. By increasing the barrier thickness to suppress the tunneling current, Levine et al. in 1990 improved the low-temperature sensitivity, and at these temperatures, the detector is suitable for detector array applications. The detectors are formally referred to as quantum well infrared photodetectors (QWIPs). In 1991, only four years after the basic detector demonstration, Bethea et al. obtained the first infrared image using a 10-element linear scanning array.

So far, the detectors have to be illuminated at an oblique angle at the edge of the mesa, since intersubband transition is only initiated by the perpendicular electric field in the radiation relative to the material layers. Additional light-coupling scheme has to be devised to create normally incident absorption, which is crucial in the 2-dimensional detector array format. In 1991, Andersson and Lundqvist proposed using diffraction gratings for this application. According to their calculations, large quantum efficiency can be obtained using proper grating parameters. Based on this coupling scheme, Bethea et al. and others have fabricated QWIPs into 2-dimensional staring arrays, and produced o high-quality and high-resolution thermal imageries. Despite these exciting achievements, oxectation, and research on an efficient flight-coupling scheme continues. Currently, there are several approaches under investigation. They can be divided into three basic approaches: the diffractive approach (e.g. the random scattering reflector and the enhanced-QWIP), the reflective approach (e.g. the corrugated-QWIP), and the refractive approach (e.g. using microlens).

Besides the light-coupling issue, there is a more serious drawback in the quantum well technology: the large thermal dark current observed at the elevated temperatures, which prevents the array to be operated at the standard temperature. In order to lower the detector dark current, Choi $e$ t al. proposed a detector structure, (referred as an infrared hot-electron transistor (IHET)), to preferentially filter out the dark current. With a proper 
filter design, the current level can be reduced arbitrarily, thus dissociating its current level from the detector cutoff wavelength. However, the detector cannot be operated with the standard photoconductor readout circuit, so a compatible readout circuit must be developed. In addition to detector applications, an IHET structure is also very useful in studying the opto-electronic properties of a QWIP. Based on these studies, detectors with better performance can be achieved.

The year of 1997 marks the tenth anniversary of the QW infrared technology. In this ten-year period, much has been learned about this technology, although some detector properties still need further studies. Since this technology reaches a certain degree of maturity, it is a suitable time to review its status. This book is designed to give a detailed and systematic discussion on the physics and the technology relevant to QWIPs. The book is organized into three parts. The first part discusses the fundamental aspects of infrared detection, and the relationship between the detector band structure and its optical absorption characteristics; the second part discusses the transport properties of the QWIP; and the third part discusses detector performance. Infrared detection is a multi-disciplinary subject, it is difficult to treat each topic with equal vigor. Nevertheless, I hope to give readers with enough background on each topic of QW infrared technology.

In addition to their impact on infrared technology, one can also witness the prevalence of quantum effects in these MQW structures. Basic premises of quantum mechanics, such as quantization in a box, quantum mechanical transmission, dipole transition, and miniband structure in superlattice, are beautifully displayed. They are, in fact, good examples of basic quantum mechanics. The ability of placing an electron with precise energy in an otherwise empty miniband can also be useful in studying several branches of physics, including band theory, theory of electron transport, hot-electron energy relaxation, noise in quantum structures, and new materials. Therefore, the study of QWIP should not be confined to infrared engineers, but should also be of interest to physicists in general. This book can serve as a resource for a course on QWIPs or as a reference in the areas of quantum or solid state physics.

I would like to express my appreciation to many of my colleagues and friends, especially Prof. R. G. Wheeler of Yale University, Prof. D. C. Tsui of Princeton University, and Dr. G. J. Iafrate of the Army Research Office, who have helped me tremendously in various stages of my career. I enjoyed collaboration with W. H. Chang, M. Dutta, M. A. Stroscio, B. F. Levine, C. G. Bethea, S. D. Gunapala, and L. Fotiadis. I would like to thank students from several universities who have participated in these research projects and produced most of the experimental results. They are W. D. Braddock, C. H. Kuan, C. Y. Lee, and C. J. Chen. I also received technical assistance from M. Taysing-Lara, G. McLane, M. Z. Tidrow, T. DeAnni, and P. Newman. 


\section{Contents}

Preface.

Acknowledgements. xi

1. Fundamentals.

1.1 Introduction ................................................................................................. 1

1.2 Blackbody radiation and flux transfer ............................................................ 4

1.2.1 Blackbody radiation......................................................................... 4

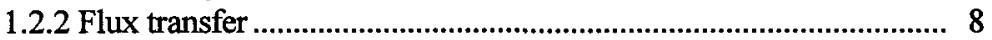

1.3 Signal and noise in photodetection................................................................ 13

1.3.1 Optical signal of a photoconductor.................................................. 14

1.3.2 Noise in a photoconductor.............................................................. 19

2. Energy Levels in One-Dimensional Quantum Wells ......................... 26

2.1 Analytical method ............................................................................................ 27

2.2 Wentzel-Kramers-Brillouin approximation...................................................... 30

2.3 Kronig-Penney model..................................................................................... 35

2.4 Transfer-matrix method ............................................................................... 54

3. Quantum Wells in Crystal Lattices .......................................................... 73

3.1 Finite in-plane momentum......................................................................... 73

3.2 Crystal symmetries and band structure of GaAs ........................................... 77

3.2.1 Basic properties of a group ……………………………………...... 77

3.2.2 Symmetry group of GaAs crystal lattice......................................... 81

$3.3 \mathrm{k} \cdot \mathrm{p}$ perturbation theory ................................................................................. 86

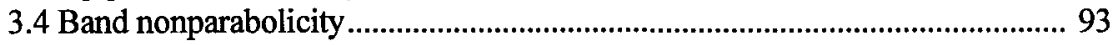

4. Quantum Wells in the Presence of Interaction ...................................... 99

4.1 Direct Coulomb interaction ........................................................................... 99

4.2 Electron-electron interaction ..........................................................................102

4.3 Electron-phonon interaction .............................................................................110

4.4 Static electric field...........................................................................................114

4.5 Oscillating electric field ................................................................................ 117

5. Intersubband Transition in Quantum wells ................................................122

5.1 Theory of dipole transition ...........................................................................122

5.2 Intersubband transition in superlattices ..........................................................125

5.3 Bound-to-bound intersubband transition..........................................................129

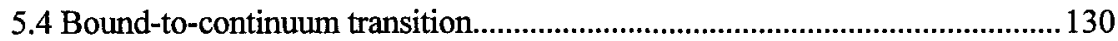

5.5 Bound-to-quasi-bound transition....................................................................143

5.6 Bound-to-miniband transition........................................................................ 144 
VIII Contents

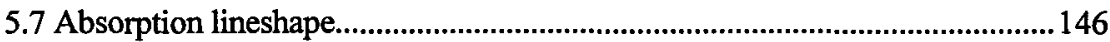

5.7.1 Bound-to-continuum transition in thick barrier quantum wells.

5.7.2 Bound-to-miniband transition in thin barrier quantum wells ........152

5.7.3 Bound-to-bound transition in thick barrier quantum wells.............155

5.8 Absorption strength

6. Optical Coupling for Quantum Well Infrared Photodetectors 159

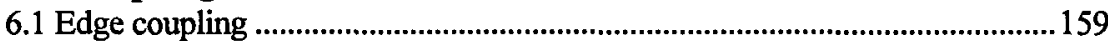

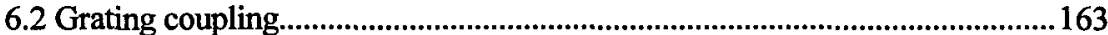

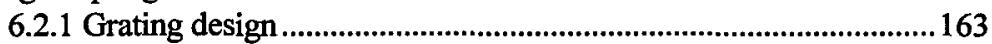

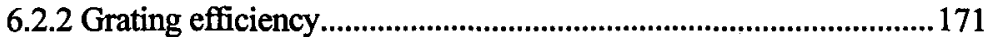

6.2.3 Grating coupled lineshape................................................................ 178

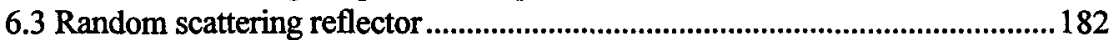

6.4 Corrugated Quantum Well Infrared Photodetectors.......................................... 189

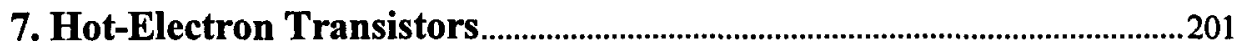

7.1 Hot-electron injection in HET ......................................................................202

7.2 Hot-electron energy relaxation..........................................................................207

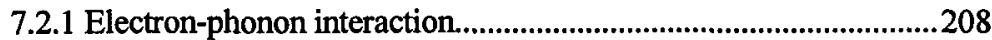

7.2.2 Electron-electron interaction........................................................215

7.2.3 Combined phonon and plasmon scattering....................................216

7.3 Experimental result from a HET .......................................................................222

7.4 Magnetophonon effect .........................................................................................226

7.5 Band nonparabolicity measured by hot-electron injection................................229

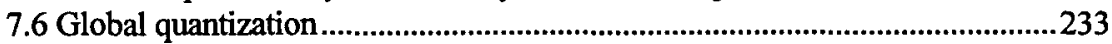

8. Excitation Hot-Electron Spectroscopy of Quantum Wells...................238

8.1 Thermally stimulated hot-electron spectroscopy...............................................238

8.1.1 Direct transport process ..................................................................241

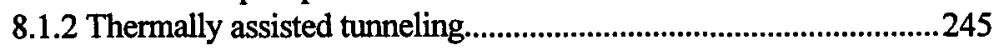

8.1.3 Thermally assisted impurity tunneling...........................................249

8.2 Optically stimulated hot-electron spectroscopy...............................................251

8.3 Electron trajectory in MQW ............................................................................254

9. Transport Properties of Multiple Quantum Well Structures..............258

9.1 Sequential resonant tunneling ..........................................................................258

9.2 Thermally assisted tunneling.........................................................................265

9.3 Thermionic miniband transport.....................................................................268

9.4 Thermal activation in multiple quantum well structures ...................................273 
10. Quantum Well Infrared Photodetector

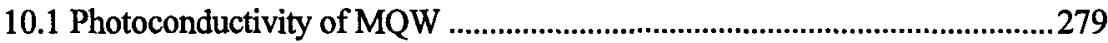

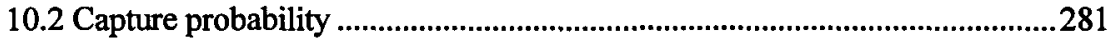

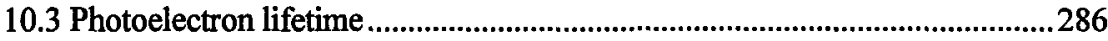

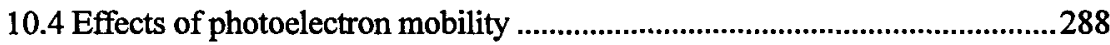

10.5 Responsivity of thin barrier MQW ...............................................................291

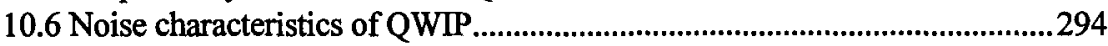

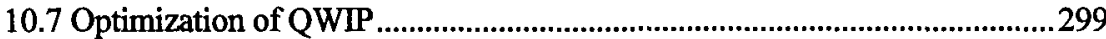

10.7.1 Detector barrier height ..................................................................300

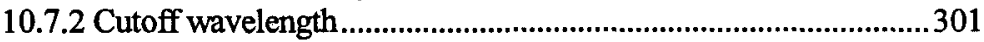

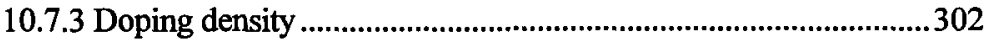

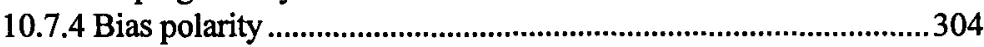

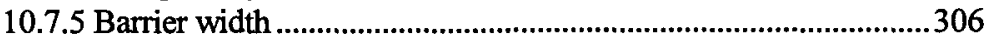

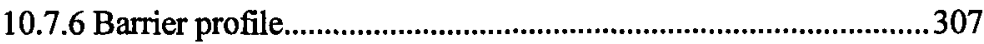

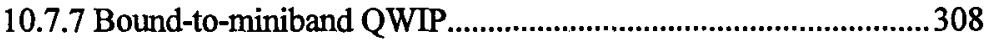

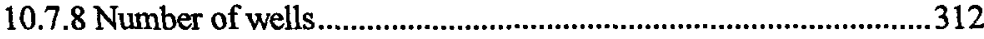

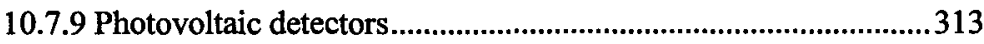

10.8 Avalanche multiplication in QWIPs .............................................................315

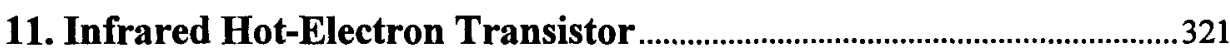

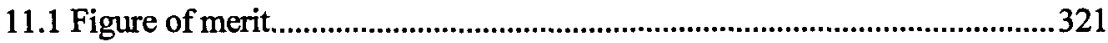

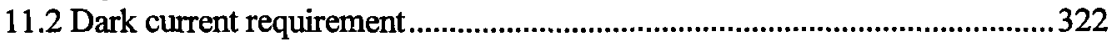

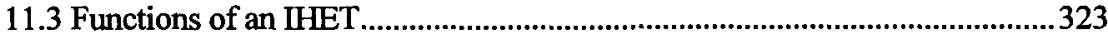

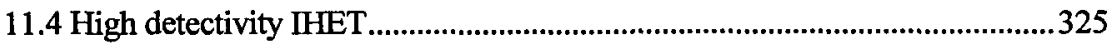

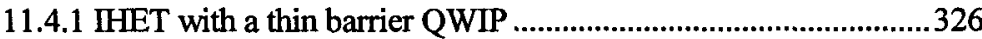

11.4.2 IHET with a thick barrier QWIP ...................................................332

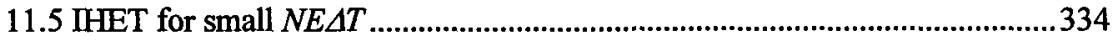

11.6 IHET for high temperature operation ...............................................................342

11.6.1 IHET with $\lambda_{c}=14 \mu \mathrm{m}$.................................................................343

11.6.2 Energy relaxation of photoelectrons in the base..........................349

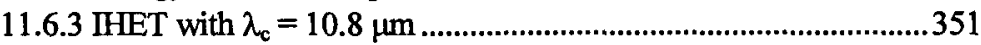

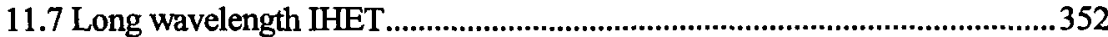

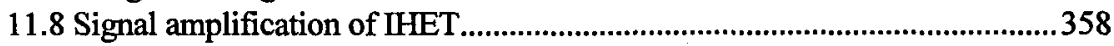

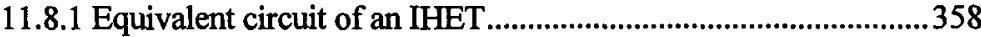

11.8.2 Experimental results......................................................................361

12. Noise Properties of Infrared Hot-Electron Transistor ........................366

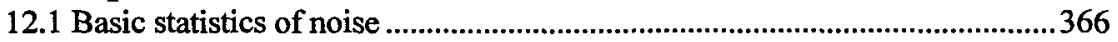

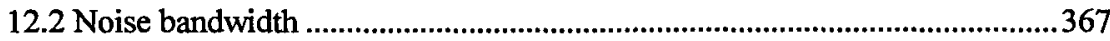

12.3 Classical noise from an IHET ...........................................................................369

12.4 Separation of g-r noise and partition noise ........................................................371

12.5 Quantum partition noise....................................................................................374 
X Contents

$12.61 / f$ noise of $Q W I P$ .380

13. Multi-Color QWIP and IHET …………....................................................382

13.1 Two-stack MQW approach ..........................................................................383

13.2 Coupled quantum well for multi-color detection..............................................385

13.3 Photoexcited coherent tunneling.....................................................................389

13.4 Thick barrier QWIP for three-color detection.................................................393

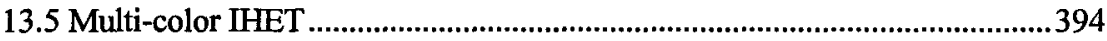

14. Performance of Quantum Well Infrared Photodetectors....................401

14.1 Background limited temperature ......................................................................401

14.2 Corrugated-QWIP for $T_{B L I P}$ improvement ....................................................406

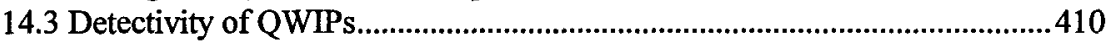

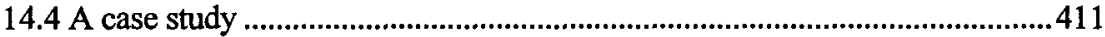

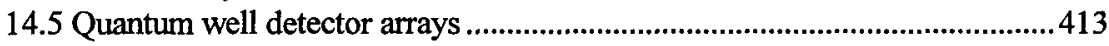

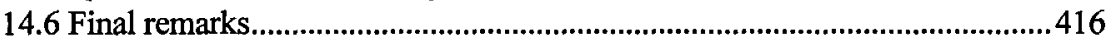

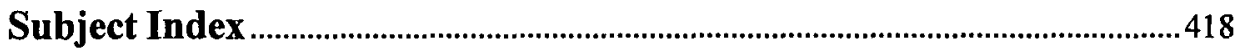




\section{Acknowledgements}

The author and publisher would like to thank the following publishers for the permission to reproduce figures:

\section{American Physical Society}

[Fig. 9.3]: Fig. 3 from Physical Review B 30, 905 (1984).

\section{IEEE Publications}

[Fig. 6.15]: Fig. 4 from IEEE Trans. on Elect. Dev 38, 1124 (1991).

\section{American Institute of Physics}

[Figs. 5.5, 5.6, 5.7, 5.8, 5.9]: Figs. 3, 4, 6, 8, 9 from J. Appl. Phys. 73, 5230 (1993).

[Fig. 5.10]: Fig. 1 from Appl. Phys. Lett. 65, 1703 (1994).

[Fig. 5.11]: Fig. 12 from J. Appl. Phys. 73, 5230 (1993).

[Figs. 5.12, 5.13, 5.20]: Figs. 1, 2, 4 from Appl. Phys. Lett. 61, 1781 (1992).

[Figs. 6.7, 6.8]: Figs. 2, 5 from Appl. Phys. Lett. 67, 1800 (1995).

[Figs. 6.10, 6.11, 6.13]: Figs. 1, 2, 3 from Appl. Phys. Lett. 64, 960 (1994).

[Figs. 6.16, 6.18, 6.20]: Figs. 1, 3, 4 from Appl. Phys. Lett. 69, 1446 (1996).

[Fig. 7.13]: Fig.1 from Appl. Phys. Lett. 68, 358 (1996).

[Figs. 7.15, 7.16]: Figs. 2, 3 from Appl. Phys. Lett. 54, 359 (1988).

[Figs. 8.3, 8.5]: Figs. 2, 4 from Appl. Phys. Lett. 57, 76 (1990).

[Figs. 8.6, 8.7]: Figs. 2, 3 from Appl. Phys. Lett. 63, 2091 (1993).

[Fig. 8.11]: Fig. 3 from Appl. Phys. Lett. 59, 3303 (1991).

[Fig. 9.2]: Fig. 2 from Appl. Phys. Lett. 60, 1324 (1992).

[Fig. 9.8]: Fig. 2 from Appl. Phys. Lett. 65, 443 (1994).

[Figs. 10.1, 10.8, 10.9]: Figs. 1, 2, 3 from J. Appl. Phys. 80, 1257 (1996).

[Fig. 10.2]: Fig. 3 from Appl. Phys. Lett. 63, 3312 (1993).

[Figs. 10.4, 10.5]: Figs. 5, 3 from Appl. Phys. Lett. 60, 592 (1992).

[Figs. 10.6, 10.7]: Figs. 5, 7 from J. Appl. Phys. 76, 1889 (1994).

[Figs. 10.19, 10.21]: Figs. 2, 1 from Appl. Phys. Lett. 51, 934 (1987).

[Fig. 11.7]: Fig. 4 from Appl. Phys. Lett. 60, 592 (1992).

[Figs. 11.11, 11.15 and 11.16, 11.17, 11.19]: Figs. 1, 2, 3, 4 from Appl. Phys. Lett. 63, 908 (1993).

[Figs. 11.23, 11.24, 11.25]: Figs. 2, 3, 4 from Appl. Phys. Lett. 66, 90 (1995). 


\section{Acknowledgements}

[Figs. 11.26, 11.27]: Figs. 3, 2 from Appl. Phys. Lett. 68, 358 (1996).

[Figs. 11.31, 11.32, 11.33, 11.35, 11.36]: Figs. 1, 2, 3, 10, 8 from J. Appl. Phys. 75, 4731 (1994).

[Fig. 11.34]: Fig. 3 from Appl. Phys. Lett. 65, 443 (1994).

[Figs. 11.39, 11.40, 11.43]: Figs. 2, 3, 4 from Appl. Phys. Lett. 59, 1614 (1991).

[Figs. 12.1, 12.3]: Figs. 1, 3 from Appl. Phys. Lett. 68, 2535 (1996).

[Figs. 12.5, 12.6]: Figs. 2, 4 from Appl. Phys. Lett. 64, 238 (1994).

[Figs. 14.1, 14.6]: Figs. 2, 3 from Appl. Phys. Lett. 65, 1703 (1994). 